\title{
Effects of coupled atomic states on the resonance scattering of radiation
}

\author{
R P Srivastavat and P R Fontana \\ Department of Physics, Oregon State University, Corvallis, Oregon 97331
}

Received 1 October 1973

\begin{abstract}
The excitation and decay probabilities for resonance scattering of radiation from an atom with two coupled excited states in an external static field are calculated as a function of time and frequency. Various oscillatory terms are found in the probabilities. These oscillations depend on the frequency of radiation, the external coupling and the energy difference between the excited states. Two special cases are investigated in detail. In the first case where one of the excited states does not decay a 'hole' appears in the emission line at a frequency equal to the frequency difference between the ground state and the unperturbed non-decaying excited state. In the second case where the two excited states decay with the same rate, one of the two lines in the emission spectrum is suppressed and the other is enhanced.
\end{abstract}

\section{Introduction}

Laser-saturated absorption methods have made it possible to eliminate the effect of Doppler broadening in resonance fluorescence processes. The technique has been used extensively to resolve spectral lines and to measure line shapes (Lee and Skolnick 1967, Barger and Hall 1969, Brewer et al 1969, Hansch et al 1971). In most resonance fluorescence calculations the details of the frequency response and the time dependence of the scattering process have been integrated out. In this paper the full frequency and time response of the resonance fluorescence process is calculated. The external perturbation couples the two excited states and its effect on the absorption and emission of radiation is investigated. The calculation gives the probability of emission of a photon with given frequency, direction and polarization for an absorbed photon with analogous specifications. In most cases of practical interest the incident light has a constant intensity over a frequency range which is large compared to the separation of the excited atomic states. This requires integration over the frequencies of the incident radiation.

When one of the excited states is non-decaying the frequency spectrum of the emitted radiation shows a characteristic 'hole' at a frequency equal to the frequency difference between the non-decaying excited state and the ground state. This hole also appears in the emitted radiation of an atom which is initially in the decaying excited state (Fontana and Srivastava 1973). For two decaying states with equal decay rates, the external perturbation enhances one of the emitted lines and suppresses the other.

† Present address : Physics Department, University of New Brunswick, Fredericton, New Brunswick, Canada. 


\section{General theory}

The resonance scattering of radiation is calculated by considering an atom which has two excited states $|a\rangle$ and $|b\rangle$ and a ground state $|c\rangle$. An external static perturbation which couples the two excited states is present. Initially, the atom is in the ground state and a beam of white light impinges on it.

The hamiltonian for this system is $\mathscr{H}=H_{0}+H+V$, where $H_{0}$ is the unperturbed hamiltonian for the atom and the radiation field, $H$ represents the interaction of the atom with the radiation field, and $V$ is the external static perturbation.

In relativistic units $(\hbar=m=c=1)$

$$
H=e \sum_{k_{\lambda}}\left(\frac{2 \pi}{L^{3} k_{\lambda}}\right)^{1 / 2}\left[a\left(k_{\lambda}\right) e^{i \boldsymbol{k} . \boldsymbol{r}}+a^{\dagger}\left(k_{\lambda}\right) \mathrm{e}^{-i \boldsymbol{k} \cdot \boldsymbol{r}}\right] \hat{\boldsymbol{e}}_{\lambda}(\boldsymbol{k}) \cdot \boldsymbol{p}
$$

where $a\left(k_{\lambda}\right)$ and $a^{\dagger}\left(k_{\lambda}\right)$ are the anihilation and creation operators, respectively, for a photon with wavevector $\boldsymbol{k}$, polarization $\lambda$, and polarization direction $\hat{\boldsymbol{e}}_{\lambda}(\boldsymbol{k})$.

It is assumed that the eigenstates of $H_{0}$ are known and thus only the effects of the perturbation $H+V$ are calculated.

In the interaction representation the state of the system at time $t$ is

$$
|\psi(t)\rangle=b_{0}(t)|0\rangle+\sum_{i} b_{i}(t)|i\rangle+\sum_{j} b_{j}(t)|j\rangle+\sum_{f} b_{f}(t)|f\rangle
$$

where the summations over $i$ and $j$ are over frequency, direction and polarization of the incident radiation and the summation over $f$ is over frequencies, directions and polarizations of the incident and emitted radiations. The state $|0\rangle$ represents the ground state, $|i\rangle$ and $|j\rangle$ the excited states with a photon absorbed and $|f\rangle$ the final states. These eigenstates are product functions of the unperturbed atomic and photon states:

$$
\begin{array}{ll}
|0\rangle=|\mathrm{c}\rangle|0\rangle_{\mathrm{rad}} ; & |i\rangle=|\mathrm{a}\rangle\left|-k_{\sigma}\right\rangle_{\mathrm{rad}} \\
|j\rangle=|\mathrm{b}\rangle\left|-k_{\sigma}\right\rangle_{\mathrm{rad}} ; \quad|f\rangle=|\mathrm{c}\rangle\left|-k_{\sigma}\right\rangle_{\mathrm{rad}}\left|k_{\lambda}\right\rangle_{\mathrm{rad}} .
\end{array}
$$

The corresponding energies are:

$$
\begin{aligned}
& E_{0}=E_{\mathrm{c}} ; \quad E_{i}=E_{\mathrm{a}}-k_{\mathrm{r}} \\
& E_{j}=-E_{\mathrm{b}}-k_{\sigma} ; \quad E_{f}=E_{\mathrm{c}}-k_{\sigma}+k_{\lambda} .
\end{aligned}
$$

The state vector $|0\rangle_{\text {rad }}$ represents the initial state of the radiation field when no photons are absorbed and $\left|-k_{\sigma}\right\rangle_{\mathrm{rad}}$ and $\left|k_{\lambda}\right\rangle_{\mathrm{rad}}$ represent the states of the radiation field with an absorbed photon with wavevector $\boldsymbol{k}_{\sigma}$, polarization $\sigma$ and polarization direction $\hat{\boldsymbol{e}}_{\sigma}$ and an emitted photon with wavevector $\boldsymbol{k}_{\lambda}$, polarization $\lambda$ and polarization direction $\hat{e}_{\lambda}$, respectively.

The differential equations for the probability amplitudes are (Heitler 1960)

$$
\mathrm{i} b_{n}(t)=\sum_{m} H_{n m}^{\prime} b_{m}(t) \mathrm{e}^{\mathrm{i}\left(E_{n}-E_{m}\right) t}+\mathrm{i} \delta_{n 0} \delta(t)
$$

where $n$ and $m$ stand for $i, j$, and $f ; H^{\prime}=H+V$ and $H_{n m}^{\prime}=\left\langle n\left|H^{\prime}\right| m\right\rangle$. The delta functions in equation (2) state the initial condition that at $t=0$ the system is in state $|0\rangle$. 
To solve equation (2), the following Fourier transformations of $b_{n}(t)$ and $\delta(t)$ are used (Heitler 1960):

$$
\begin{aligned}
& b_{n}(t)=-\frac{1}{2 \pi \mathrm{i}} \int_{-\infty}^{\infty} \mathrm{d} E G_{n}(E) \mathrm{e}^{\mathrm{i}\left(E_{n}-E\right) t} \\
& \mathrm{i} \delta(t)=-\frac{1}{2 \pi \mathrm{i}} \int_{-\infty}^{\infty} \mathrm{d} E \mathrm{e}^{\mathrm{i}\left(E_{0}-E\right) t}
\end{aligned}
$$

Substituting these expressions into equation (2) gives

$$
\left(E-E_{n}\right) G_{n}(E)=\sum_{m} H_{n m}^{\prime} G_{m}(E)+\delta_{n 0} .
$$

If one neglects two photon processes and higher order effects then

$$
\begin{aligned}
& H_{f 0}^{\prime}=H_{0 f}^{\prime}=0 \\
& H_{i i^{\prime}}^{\prime}=V_{\mathrm{aa}} \delta_{k_{\sigma} k_{\sigma}^{\prime}} \quad H_{j j^{\prime}}^{\prime}=V_{\mathrm{bb}} \delta_{k_{\sigma} k_{\sigma}^{\prime}} \\
& H_{f f^{\prime}}^{\prime}=V_{\mathrm{cc}} \delta_{k_{\sigma} k_{\sigma}^{\prime}} \delta_{k_{\lambda} k_{\lambda}^{\prime}} \quad H_{i j^{\prime}}^{\prime}=V_{\mathrm{ab}} \delta_{k_{\sigma} k_{\sigma}^{\prime}} \\
& H_{0 \mathrm{i}}^{\prime}=H_{0 \mathrm{i}} \quad H_{0 j}^{\prime}=H_{0 j} \quad H_{\mathrm{i} f^{\prime}}^{\prime}=H_{\mathrm{i} f^{\prime}} \delta_{k_{\sigma} k_{\sigma}} \\
& H_{j f^{\prime}}^{\prime}=H_{j f^{\prime}} \delta_{k_{\sigma} k_{\sigma}^{\prime}}
\end{aligned}
$$

where

$$
V_{l m}=\langle l|V| m\rangle \text {. }
$$

Substituting these matrix elements into equation (5), one gets

$$
\begin{aligned}
& \left(E-E_{0}^{\prime}\right) G_{0}(E)=\sum_{i} H_{0 i} G_{i}(E)+\sum_{j} H_{0 j} G_{j}(E)+1 \\
& \left(E-E_{i}^{\prime}\right) G_{i}(E)=H_{i 0} G_{0}(E)+V_{\mathrm{ab}} G_{j}(E)+\sum_{f} H_{i f} G_{f}(E) \\
& \left(E-E_{j}^{\prime}\right) G_{j}(E)=H_{j 0} G_{0}(E)+V_{\mathrm{ba}} G_{i}(E)+\sum_{j} H_{j f} G_{f}(E) \\
& \left(E-E_{f}^{\prime}\right) G_{f}(E)=H_{f i} G_{i}(E)+H_{f j} G_{j}(E)
\end{aligned}
$$

where

$$
\begin{aligned}
& E_{0}^{\prime}=E_{\mathrm{c}}+V_{\mathrm{cc}} ; \quad E_{i}^{\prime}=E_{i}+V_{\mathrm{aa}} ; \quad E_{j}^{\prime}=E_{j}+V_{\mathrm{bb}} \\
& E_{f}^{\prime}=E_{f}+V_{\mathrm{cc}} .
\end{aligned}
$$

The summation over $f$ is over frequency, direction, and polarization of the emitted radiation only, since the matrix elements $H_{i f}$ and $H_{j f}$ are zero if the state $|f\rangle$ contains an absorbed photon which differs from the photon in states $|i\rangle$ and $|j\rangle$, respectively.

The equations for the Fourier coefficients can be solved by substituting $G_{f}$ from equation (11) into equations (9)-(10). This procedure eliminates the final states and produces a set of equations which are the Fourier transforms of the Weisskopf and Wigner (1930) equations.

By defining

$$
\begin{aligned}
& -\frac{\mathrm{i}}{2} \gamma_{i j}=\sum_{f} \frac{H_{i f} H_{f j}}{E-E_{f}^{\prime}} \\
& V_{i j}^{\prime}=V_{i j}-\frac{\mathrm{i}}{2} \gamma_{i j}
\end{aligned}
$$


and

$$
\begin{aligned}
-\frac{\mathrm{i}}{2} \Gamma(E)=\sum_{k_{\sigma}} & {\left[H_{0 i} V_{i j}^{\prime} H_{j 0}+H_{j 0} V_{j i}^{\prime} H_{i 0}+\left|H_{0 i}\right|^{2}\left(E-E_{j}^{\prime}+(\mathrm{i} / 2) \gamma_{j j}\right)+\left|H_{0 j}\right|^{2}\left(E-E_{i}^{\prime}+(\mathrm{i} / 2) \gamma_{i i}\right)\right] } \\
& \times\left[\left(E-E_{i}^{\prime}+(\mathrm{i} / 2) \gamma_{i i}\right)\left(E-E_{j}^{\prime}+(\mathrm{i} / 2) \gamma_{j j}\right)-V_{i j}^{\prime} V_{j i}^{\prime}\right]^{-1}
\end{aligned}
$$

one gets the following expressions for the Fourier coefficients:

$$
\begin{aligned}
G_{0}(E) & =\frac{1}{E-E_{0}^{\prime}+(\mathrm{i} / 2) \Gamma(E)} \\
G_{i}(E) & =\frac{\left[H_{i 0}\left(E-E_{j}^{\prime}+(\mathrm{i} / 2) \gamma_{j j}(E)\right)+V_{i j}^{\prime} H_{j 0}\right] G_{0}(E)}{\left(E-E_{i}^{\prime}+(\mathrm{i} / 2) \gamma_{i i}\right)\left(E-E_{j}^{\prime}+(\mathrm{i} / 2) \gamma_{j j}\right)-V_{i j}^{\prime} V_{j i}^{\prime}} \\
G_{j}(E) & =\frac{\left[H_{j 0}\left(E-E_{i}^{\prime}+(\mathrm{i} / 2) \gamma_{i i}(E)\right)+V_{j i}^{\prime} H_{i 0}\right] G_{0}(E)}{\left(E-E_{i}^{\prime}+(\mathrm{i} / 2) \gamma_{i i}\right)\left(E-E_{j}^{\prime}+(\mathrm{i} / 2) \gamma_{j j}\right)-V_{i j}^{\prime} V_{j i}^{\prime}}
\end{aligned}
$$

and

$$
G_{f}(E)=\zeta\left(E-E_{f}^{\prime}\right)\left[H_{f i} G_{i}(E)+H_{f j} G_{j}(E)\right] .
$$

It can be shown that the $\gamma_{i j}$ for $i \neq j$ vanish whenever $|i\rangle$ and $|j\rangle$ are states of good angular momentum (Breit 1933, Himmell 1965). It is assumed that this is the case here. In addition the real parts of $\gamma_{i i}(E)$ and $\gamma_{j j}(E)$ can be replaced by the real parts of $\gamma_{i i}\left(E_{i}^{\prime}\right)$ and $\gamma_{j j}\left(E_{j}^{\prime}\right)$, respectively, and the real part of $\Gamma(E)$ is almost independent of $E$ and very small compared to the real parts of $\gamma_{i i}\left(E_{i}^{\prime}\right)$ and $\gamma_{j j}\left(E_{j}^{\prime}\right)$, provided the incident beam is not too intense (Srivastava 1972). The imaginary parts of $\gamma_{i i}\left(E_{i}^{\prime}\right), \gamma_{j j}\left(E_{j}^{\prime}\right)$, and $\Gamma(E)$ can be absorbed into $E_{i}, E_{j}$, and $E_{0}$, respectively, giving rise to energy shifts (Bethe and Salpeter 1957, Heitler 1960). Thus by defining

$$
\Gamma=\operatorname{Re}\{\Gamma(E)\} \quad \gamma_{\mathrm{a}}=\operatorname{Re}\left\{\gamma_{i i}\left(E_{i}^{\prime}\right)\right\}
$$

and

$$
\gamma_{\mathrm{b}}=\operatorname{Re}\left\{\gamma_{j j}\left(E_{j}^{\prime}\right)\right\}
$$

the expressions for the Fourier coefficients reduce to

$$
\begin{aligned}
G_{0}(E) & =\frac{1}{E-E_{0}^{\prime}+(\mathrm{i} / 2) \Gamma} \\
G_{i}(E) & =\frac{\left[H_{i 0}\left(E-E_{j}^{\prime}+(\mathrm{i} / 2) \gamma_{\mathrm{b}}\right)+V_{\mathrm{ab}} H_{j 0}\right] G_{0}}{\left(E-E_{i}^{\prime}+(\mathrm{i} / 2) \gamma_{\mathrm{a}}\right)\left(E-E_{j}^{\prime}+(\mathrm{i} / 2) \gamma_{\mathrm{b}}\right)-\left|V_{\mathrm{ab}}\right|^{2}} \\
G_{j}(E) & =\frac{\left[H_{j 0}\left(E-E_{i}^{\prime}+(\mathrm{i} / 2) \gamma_{\mathrm{a}}\right)+V_{\mathrm{ba}} H_{i 0}\right] G_{0}}{\left(E-E_{i}^{\prime}+(\mathrm{i} / 2) \gamma_{\mathrm{a}}\right)\left(E-E_{j}^{\prime}+(\mathrm{i} / 2) \gamma_{\mathrm{b}}\right)-\left|V_{\mathrm{ab}}\right|^{2}} .
\end{aligned}
$$

The Fourier coefficient $G_{f}(E)$ (equation (17)) has four poles at energies

$$
\begin{aligned}
& E_{1}^{\prime}=\left(E_{\mathrm{a}}^{\prime}+E_{\mathrm{b}}^{\prime}\right) / 2-k_{\sigma}-\mathrm{i}\left(\gamma_{\mathrm{a}}+\gamma_{\mathrm{b}}\right) / 4+(R+\mathrm{i} I) \\
& E_{2}^{\prime}=\left(E_{\mathrm{a}}^{\prime}+E_{\mathrm{b}}^{\prime}\right) / 2-k_{\sigma}-\mathrm{i}\left(\gamma_{\mathrm{a}}+\gamma_{\mathrm{b}}\right) / 4-(R+\mathrm{i} I) \\
& E_{3}^{\prime}=E_{0}^{\prime}-(\mathrm{i} / 2) \Gamma \\
& E_{4}^{\prime}=E_{f}^{\prime}
\end{aligned}
$$


where

$$
\begin{aligned}
R+i I=\frac{1}{2 \sqrt{ } 2}\left[\left\{\left(\Delta^{2}-X^{2}+4 V^{2}\right)^{2}+4 \Delta^{2} X^{2}\right\}^{1 / 2}+\left(\Delta^{2}-X^{2}+4 V^{2}\right)\right]^{1 / 2} \\
-\frac{\mathrm{i}}{2 \sqrt{2}}\left[\left\{\left(\Delta^{2}-X^{2}+4 V^{2}\right)^{2}+4 \Delta^{2} X^{2}\right\}^{1 / 2}-\left(\Delta^{2}-X^{2}+4 V^{2}\right)\right]^{1 / 2}
\end{aligned}
$$

and

$$
\begin{aligned}
& \Delta=E_{\mathrm{a}}^{\prime}-E_{\mathrm{b}}^{\prime} \\
& X=\left(\gamma_{\mathrm{a}}-\gamma_{\mathrm{b}}\right) / 2 \\
& V^{2}=\left|V_{\mathrm{ab}}\right|^{2} .
\end{aligned}
$$

In equation (20) $R$ is the real part and $I$ the imaginary part of the expression. The real part produces an energy shift of the atomic levels and the imaginary part determines the decay characteristics. For $\Delta \neq 0$ the first term in equation (20) is real, but for $\Delta=0$ it is real only if $4 V^{2} \geqslant X^{2}$ and is pure imaginary if $4 V^{2}<X^{2}$. For $\Delta=0$ or $X=0$ the second term in equation (20) vanishes for any $V$. For $\Delta=0$ and for $V \leqslant \frac{1}{2}|X|$ the perturbation does not produce an energy shift, and for large $V$ the energy shift becomes proportional to $V$. For $X=0\left(\gamma_{\mathrm{a}}=\gamma_{\mathrm{b}}\right)$ the energy shifts of the atomic states are the same as the ones obtained from a time-independent perturbation calculation, taking only the static perturbation into account (Bates 1961). For large values of $X$, however, the radiation damping affects the energy shifts significantly. The radiative interaction diminishes the effect of the static perturbation. For $V=0$ the imaginary part $I$ is independent of $\Delta$, and for $\Delta=0, I$ vanishes for $V \geqslant \frac{1}{2}|X|$.

The probability amplitudes $b_{n}(t)$ are calculated by evaluating the integrals in equation (3) by contour integration. The expressions for the Fourier coefficients $G_{n}$ are given in equation (17) and equation (18). Since the imaginary parts of the poles of $G_{n}$ are negative, the path of integration is taken to be a clockwise infinite semicircular contour in the lower half of the complex plane. The zeta function in $G_{f}$ causes the path of integration for $b_{f}(t)$ along the real axis to go around a semicircle of infinitely small radius centred at $E=E_{f}^{\prime}$. These integrations yield

$$
\begin{gathered}
b_{0}(t)=\mathrm{e}^{-\mathrm{i} V_{\mathrm{co} t}-\Gamma t / 2} \\
b_{i}(t)=\frac{H_{i 0}\left(E_{1}^{\prime}-E_{j}^{\prime}+(\mathrm{i} / 2) \gamma_{\mathrm{b}}\right)+V_{\mathrm{ab}} H_{j 0}}{\left(E_{1}^{\prime}-E_{2}^{\prime}\right)\left(E_{1}^{\prime}-E_{3}^{\prime}\right)} \mathrm{e}^{\mathrm{i}\left(E_{\mathrm{i}}-E_{1}^{\prime}\right) t} \\
+\frac{H_{i 0}\left(E_{2}^{\prime}-E_{j}^{\prime}+(\mathrm{i} / 2) \gamma_{\mathrm{b}}\right)+V_{\mathrm{ab}} H_{j 0}}{\left(E_{2}^{\prime}-E_{1}^{\prime}\right)\left(E_{2}^{\prime}-E_{3}^{\prime}\right)} \mathrm{e}^{\mathrm{i}\left(E_{\mathrm{i}}-E_{2}^{\prime}\right) t} \\
b_{j}(t)=\frac{H_{j 0}\left(E_{1}^{\prime}-E_{i}^{\prime}+(\mathrm{i} / 2) \gamma_{\mathrm{a}}\right)+V_{\mathrm{ba}} H_{i 0}}{\left(E_{1}^{\prime}-E_{2}^{\prime}\right)\left(E_{1}^{\prime}-E_{3}^{\prime}\right)} \mathrm{e}^{\mathrm{i}\left(E_{j}-E_{1}^{\prime}\right) t} \\
+\frac{H_{j 0}\left(E_{2}^{\prime}-E_{i}^{\prime}+(\mathrm{i} / 2) \gamma_{\mathrm{a}}\right)+V_{\mathrm{ba}} H_{i 0}}{\left(E_{2}^{\prime}-E_{1}^{\prime}\right)\left(E_{2}^{\prime}-E_{3}^{\prime}\right)} \mathrm{e}^{\mathrm{i}\left(E_{j}-E_{2}^{\prime}\right) t} \\
+\frac{H_{j 0}\left(E_{3}^{\prime}-E_{i}^{\prime}+(\mathrm{i} / 2) \gamma_{\mathrm{a}}\right)+V_{\mathrm{ba}} H_{i 0}}{\left(E_{3}^{\prime}-E_{1}^{\prime}\right)\left(E_{3}^{\prime}-E_{2}^{\prime}\right)} \mathrm{e}^{\mathrm{i}\left(E_{j}-E_{3}^{\prime}\right) t}
\end{gathered}
$$


and

$$
\begin{gathered}
b_{f}(t)=\sum_{l}\left(\left[H_{f i}\left\{H_{i 0}\left(E_{l}^{\prime}-E_{j}^{\prime}+(\mathrm{i} / 2) \gamma_{\mathrm{b}}\right)+V_{\mathrm{ab}} H_{j 0}\right\}+H_{f j}\left\{H_{j 0}\left(E_{l}^{\prime}-E_{i}^{\prime}+\mathrm{i}\left(\gamma_{\mathrm{a}} / 2\right)\right)+V_{\mathrm{ba}} H_{i 0}\right\}\right]\right. \\
\left.\times \frac{\mathrm{e}^{\mathrm{i}\left(E_{f}-E_{i}^{\prime}\right) t}}{\Pi_{m}\left(E_{l}^{\prime}-E_{m}^{\prime}\right)}\right) \quad m \neq l
\end{gathered}
$$

where $l$ and $m$ varies over the subscripts $f, 1,2$, and 3 .

The probability $\left|b_{0}(t)\right|^{2}$ decays monotonically with a single decay constant $\Gamma$. The probabilities $\left|b_{i}(t)\right|^{2}$ and $\left|b_{j}(t)\right|^{2}$ have three pure exponentially decaying terms with different decay constants and three terms with modulated decay. The modulation frequencies are different for different terms in each probability and so are the decay constants. The modulation frequencies depend on the frequencies of the absorbed photons, the external perturbation $V$, and the energy difference between the excited states. The decay constants also depend on $V$ and the energy difference $\Delta$. The probability $\left|b_{f}(t)\right|^{2}$ has four exponentially decaying terms and six terms with modulated decay. These modulation frequencies and the decay constants are similar to the ones in $\left|b_{i}(t)\right|^{2}$ and $\left|b_{j}(t)\right|^{2}$.

As $t \rightarrow \infty$, only the first term in $b_{f}(t)$ survives and all the other probability amplitudes decay to zero. The probability $\left|b_{f}(\infty)\right|^{2}$ is the probability that a photon with wavevector $\boldsymbol{k}_{\sigma}$ and polarization vector $\hat{\boldsymbol{e}}_{\sigma}$ has been absorbed and a photon with wavevector $\boldsymbol{k}_{\lambda}$ and polarization vector $\hat{\boldsymbol{e}}_{z}$, has been emitted.

To get the probability of emission of a photon one has to sum over the incident frequencies

$$
P\left(\Omega_{\mathrm{i}}, \hat{\boldsymbol{e}}_{\sigma}, \boldsymbol{k}_{\lambda}\right)=\sum_{k_{\sigma}}\left|b_{f}(\infty)\right|^{2}
$$

where the summation over $k_{\sigma}$ is over the frequencies of the absorbed photons only. The direction of the incident beam is specified by $\Omega_{\mathrm{i}}$.

For a continuous distribution of the incident frequencies, the summation over $k_{\sigma}$ in equation (26) is replaced by an integration over $k_{\sigma}$. In general, $P\left(\Omega_{i}, \hat{\boldsymbol{e}}_{\sigma}, \boldsymbol{k}_{\lambda}\right)$ has two peaks. The lineshapes and linewidths depend on the external static perturbation, the energy separation of the excited states, and the decay constants of the excited states.

The intensity of the scattered radiation $I\left(\Omega_{\mathrm{i}}, \Omega_{\mathrm{s}}, \hat{\boldsymbol{e}}_{\sigma}, \hat{\boldsymbol{e}}_{\hat{\lambda}}\right)$ for given directions and polarizations of the incident and the scattered beams is obtained by integrating the probability $P\left(\Omega_{i}, \hat{\boldsymbol{e}}_{\sigma}, \boldsymbol{k}_{\lambda}\right)$ over the frequency $k_{\lambda}$ :

$$
I\left(\Omega_{\mathrm{i}}, \Omega_{\mathrm{s}}, \hat{\boldsymbol{e}}_{\mathrm{r}}, \hat{\boldsymbol{e}}_{\lambda}\right)=(L / 2 \pi)^{3} \int_{0}^{\infty} P\left(\Omega_{\mathrm{i}}, \hat{\boldsymbol{e}}_{\sigma}, \boldsymbol{k}_{\lambda}\right) \boldsymbol{k}_{\lambda}^{2} \mathrm{~d} k_{\lambda}
$$

where $\Omega_{\mathrm{s}}$ indicates the direction of the scattered beam.

\section{Resonance fluorescence with one decaying and one non-decaying state}

The theory developed in $\S 2$ is used here to discuss the resonance scattering of radiation from an atomic system where only one of the excited states is decaying. The state $|b\rangle$ is considered to be the non-decaying state $\left(\gamma_{\mathrm{b}}=0\right)$. 
The real parts of the poles of the Fourier coefficients give the perturbed energies of the system. From equation (19), the energies of the two perturbed excited states are:

$$
\left.\begin{array}{l}
\operatorname{Re}\left(E_{1}^{\prime}\right) \\
\operatorname{Re}\left(E_{2}^{\prime}\right)
\end{array}\right\}=\left(E_{\mathrm{a}}^{\prime}+E_{\mathrm{b}}^{\prime}\right) / 2 \pm R
$$

where $R$ is the real part of equation (20) with $X=\gamma_{\mathrm{a}} / 2$.

The probability amplitudes are obtained from equation (22) to equation (25) by setting $\gamma_{b}, H_{j 0}$ and $H_{f j}$ equal to zero. The results are:

$$
\begin{gathered}
b_{0}(t)=\mathrm{e}^{-\mathrm{i} V_{c o} c^{t}-\Gamma t / 2} \\
b_{i}(t)=\frac{H_{i 0}\left(E_{1}^{\prime}-E_{j}^{\prime}\right)}{\left(E_{1}^{\prime}-E_{2}^{\prime}\right)\left(E_{1}^{\prime}-E_{3}^{\prime}\right)} \mathrm{e}^{\mathrm{i}\left(E_{i}-E_{1}^{\prime}\right) t}+\frac{H_{i 0}\left(E_{2}^{\prime}-E_{j}^{\prime}\right)}{\left(E_{2}^{\prime}-E_{1}^{\prime}\right)\left(E_{2}^{\prime}-E_{3}^{\prime}\right)} \mathrm{e}^{\mathrm{i}\left(E_{i}-E_{2}^{\prime}\right) t} \\
\quad+\frac{H_{i 0}\left(E_{3}^{\prime}-E_{j}^{\prime}\right)}{\left(E_{3}^{\prime}-E_{1}^{\prime}\right)\left(E_{3}^{\prime}-E_{2}^{\prime}\right)} \mathrm{e}^{\mathrm{i}\left(E_{i}-E_{3}^{\prime}\right) t} \\
b_{j}(t)=\frac{V_{\mathrm{ba}} H_{i 0}}{\left(E_{3}^{\prime}-E_{1}^{\prime}\right)\left(E_{3}^{\prime}-E_{2}^{\prime}\right)} \mathrm{e}^{\mathrm{i}\left(E_{j}-E_{3}^{\prime}\right) t}+\frac{V_{\mathrm{ba}_{\mathrm{a}} H_{i 0}}}{E_{1}^{\prime}-E_{2}^{\prime}}\left[\frac{\mathrm{e}^{\mathrm{i}\left(E_{j}-E_{1}\right) t}}{\left(E_{1}^{\prime}-E_{3}^{\prime}\right)}-\frac{\mathrm{e}^{\mathrm{i}\left(E_{j}-E_{2}^{\prime}\right) t}}{\left(E_{2}^{\prime}-E_{3}^{\prime}\right)}\right]
\end{gathered}
$$

and

$$
\begin{gathered}
b_{f}(t)=\frac{H_{f i} H_{i 0}\left(E_{f}^{\prime}-E_{j}^{\prime}\right)}{\left(E_{f}^{\prime}-E_{1}^{\prime}\right)\left(E_{f}^{\prime}-E_{2}^{\prime}\right)\left(E_{f}^{\prime}-E_{3}^{\prime}\right)} \mathrm{e}^{-\mathrm{i} V_{\mathrm{cc} t}}+\frac{H_{f i} H_{i 0}\left(E_{1}^{\prime}-E_{j}^{\prime}\right)}{\left(E_{1}^{\prime}-E_{f}^{\prime}\right)\left(E_{1}^{\prime}-E_{2}^{\prime}\right)\left(E_{1}^{\prime}-E_{3}^{\prime}\right)} \mathrm{e}^{\mathrm{i}\left(E_{f}-E_{1}^{\prime}\right) t} \\
+\frac{H_{f i} H_{i 0}\left(E_{2}^{\prime}-E_{j}^{\prime}\right)}{\left(E_{2}^{\prime}-E_{1}^{\prime}\right)\left(E_{2}^{\prime}-E_{f}^{\prime}\right)\left(E_{2}^{\prime}-E_{3}^{\prime}\right)} \mathrm{e}^{\mathrm{i}\left(E_{f}-E_{2}^{\prime}\right) t} \\
\quad+\frac{H_{f i} H_{i 0}\left(E_{3}^{\prime}-E_{j}^{\prime}\right)}{\left(E_{3}^{\prime}-E_{f}^{\prime}\right)\left(E_{3}^{\prime}-E_{1}^{\prime}\right)\left(E_{3}^{\prime}-E_{2}^{\prime}\right)} \mathrm{e}^{\mathrm{i}\left(E_{f}-E_{3}^{\prime}\right) t}
\end{gathered}
$$

The energies $E_{1}^{\prime}, E_{2}^{\prime}$, and $E_{3}^{\prime}$ are defined in equation (19).

From equation (29), one finds that the probability $\left|b_{0}(t)\right|^{2}$ of the ground state decays exponentially with a decay constant $\Gamma$.

The probability $\left|b_{i}(t)\right|^{2}$ gives the probability of the atom being in the excited state |a) with an absorbed photon with wave vector $\boldsymbol{k}_{\sigma}$. This probability has three pure decaying terms with decay constants $\left(\gamma_{\mathrm{a}} / 2-2 I\right),\left(\gamma_{\mathrm{a}} / 2+2 I\right)$ and $\Gamma$ and three oscillatory terms with decaying amplitudes. The oscillation frequencies are $2 R, K_{\sigma}+R$ and $K_{\sigma}-R$ and the decay constants of the amplitudes are, respectively, $\gamma_{a} / 2,\left(\gamma_{\mathrm{a}} / 4+I+\Gamma / 2\right)$ and $\left(\gamma_{\mathrm{a}} / 4-I+\Gamma / 2\right)$, where $R$ and $I$ are defined in equation $(20)$ and $K_{\sigma}$ as

$$
K_{\sigma}=k_{\sigma}-\left(E_{\mathrm{a}}^{\prime}+E_{\mathrm{b}}^{\prime}\right) / 2+E_{\mathrm{c}}^{\prime} .
$$

The probability $\left|b_{i}(t)\right|^{2}$ is plotted in figure 1 as a function of $K_{\sigma}$ for different times. At short times, this probability is small and quite broad, but as the time increases, the probability narrows up into two peaks, one near $K_{\sigma}=R$ and the other near $K_{\sigma}=-R$. The peak near $K_{\sigma}=-R$ is weaker than the peak near $K_{\sigma}=R$. At small times, the height of the peaks increases for some time and then starts decreasing at larger times and becomes zero as $t \rightarrow \infty$. For intermediate times an increase in $V$ decreases the height of the peak near $K_{\sigma}=R$ and increases the height of the peak near $K_{\sigma}=-R$. 


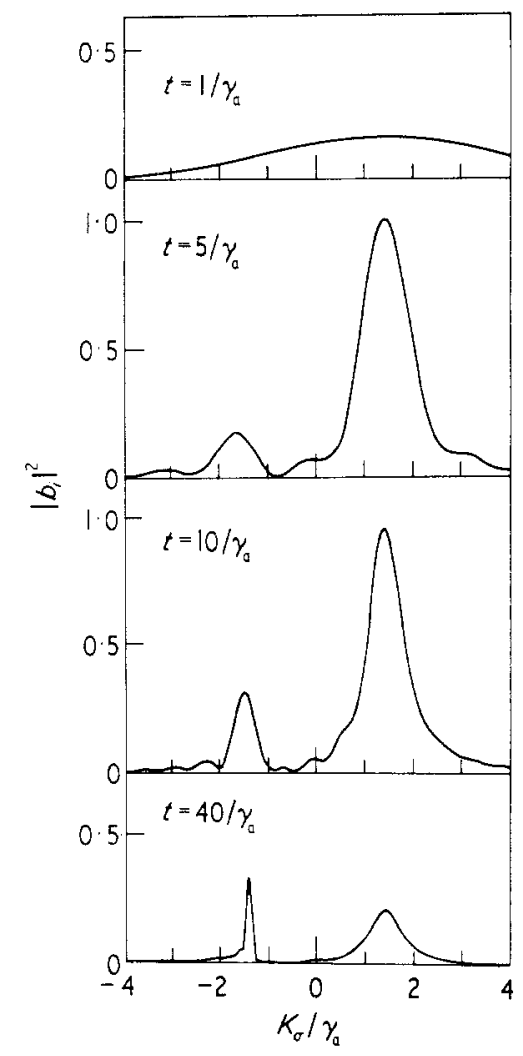

Figure 1. Probability $\left|b_{i}(t)\right|^{2}$ as a function of $K_{\sigma}$ for the case where one of the excited states is non-decaying $\left(\gamma_{b}=0\right)$. The energy difference $\Delta$ is $2 \gamma_{a}, \Gamma$ is equal to $0.05 \gamma_{a}$ and the external static perturbation $V$ is $\gamma_{a}$. The origin of the $K_{\sigma}$ axis is at the energy difference between the ground state and the average energy of the excited states.

For small $V$ the perturbed state near $K_{\sigma}=-R$ has only a small admixture of the decaying state $|\mathrm{a}\rangle$, whereas the perturbed state near $K_{\sigma}=R$ is almost identical with state $|\mathrm{a}\rangle$. As $V$ increases, the probability of the atom in state $|a\rangle$ decreases near $K_{\sigma}=R$ and increases near $K_{\sigma}=-R$, and for large $V$ the two peaks have the same height.

The probability $\left|b_{j}(t)\right|^{2}$ has also three pure decaying terms and three oscillatory terms with decaying amplitudes. The oscillation frequencies and decay constants are the same as those obtained in the case of $\left|b_{i}(t)\right|^{2}$.

The probability $\left|b_{f}(t)\right|^{2}$ gives the probability of the atom in the state $|c\rangle$ with a photon with wavevector $\boldsymbol{k}_{\sigma}$ and polarization direction $\hat{\boldsymbol{e}}_{\sigma}$ absorbed, and a photon with wavevector $\boldsymbol{k}_{\lambda}$ and polarization direction $\hat{\boldsymbol{e}}_{\lambda}$ emitted. This probability is plotted in figure 2 as a function of the absorbed frequency $k_{\sigma}$ for a given emitted frequency $k_{\lambda}$ and given time $t$. One principal maximum is observed at $k_{\sigma}=k_{\lambda}$ with many secondary maxima. The principal maxima are wide for short times and narrow up at large times. This means that the off channel $\left(k_{\sigma} \neq k_{\lambda}\right)$ scattering is quite significant at small times but becomes negligible at large times. As $t \rightarrow \infty$, the plot of $\left|b_{f}(t)\right|^{2}$ as a function of $K_{\sigma}$ becomes a Dirac delta function $\delta\left(k_{\sigma}-k_{\lambda}\right)$.

The probability $P\left(\Omega_{\mathrm{i}}, \hat{\boldsymbol{e}}_{\sigma}, \boldsymbol{k}_{\lambda}\right)$ that a photon with wavevector $\boldsymbol{k}_{\lambda}$ and polarization direction $\hat{\boldsymbol{e}}_{\lambda}$ has been emitted between time $t=0$ and $t \rightarrow \infty$, is obtained from equation 


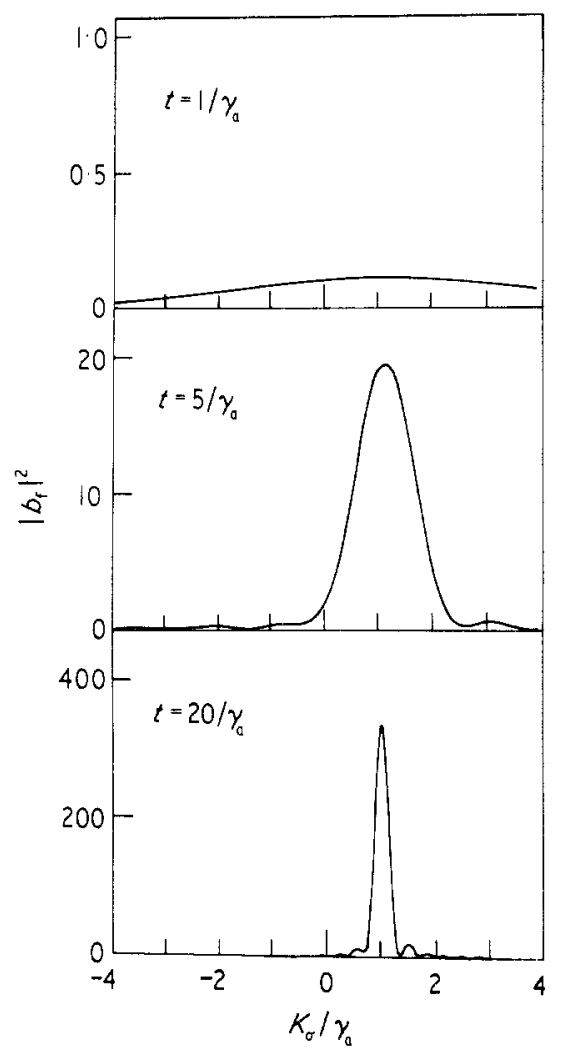

Figure 2. Probability $\left|b_{f}(t)\right|^{2}$ as a function of $K_{\sigma}$ for $K_{\lambda}=\gamma_{\mathrm{a}}, \gamma_{\mathrm{b}}=0, \Delta=2 \gamma_{\mathrm{a}}, \Gamma=0.05 \gamma_{\mathrm{a}}$ and $V=\gamma_{\mathrm{a}}$. The origin of the $K_{\sigma}$ axis is at the energy difference between the ground state and the average energy of the excited states.

(26). The result is

$$
P\left(\Omega_{\mathrm{i}}, \hat{\boldsymbol{e}}_{\sigma}, \boldsymbol{k}_{\lambda}\right)=\frac{\left[e^{4} I_{0} /\left(L^{3} \Gamma k_{0}^{3}\right)\right]\left[\left.P_{\mathrm{ca}}^{i}\right|^{2}\left|P_{\mathrm{ac}}^{\sigma}\right|^{2}(K+\Delta / 2)^{2}\right.}{\left[(K-\Delta / 2)(K+\Delta / 2)-V^{2}\right]^{2}+(K+\Delta / 2)^{2} \gamma_{\mathrm{a}}^{2} / 4}
$$

where

$$
K=k_{\lambda}-\left(E_{\mathrm{a}}^{\prime}+E_{\mathrm{b}}^{\prime}\right) / 2+E_{\mathrm{c}}^{\prime}
$$

and the matrix elements $P_{\mathrm{c} m}^{l}$ are defined as

$$
P_{\mathrm{c} m}^{l}=\left\langle\mathrm{c}\left|\boldsymbol{p} \cdot \hat{\boldsymbol{e}}_{\mid}\right| m\right\rangle ; \quad m=\mathrm{a}, \mathrm{b} ; l=\lambda, \sigma .
$$

This probability has two maxima, one at $K=\frac{1}{2}\left(\Delta^{2}+4 V^{2}\right)^{1 / 2}$ and the other at $K=-\frac{1}{2}\left(\Delta^{2}+4 V^{2}\right)^{1 / 2}$. The maxima have equal heights but unequal widths. These widths are given by

$$
\omega_{1}=\gamma_{\mathrm{a}} / 2+\frac{1}{2}\left[\left(\gamma_{\mathrm{a}}^{2} / 4+\Delta^{2}+4 V^{2}+\gamma_{\mathrm{a}} \Delta\right)^{1 / 2}-\left(\gamma_{\mathrm{a}}^{2} / 4+\Delta^{2}+4 V^{2}-\gamma_{\mathrm{a}} \Delta\right)^{1 / 2}\right]
$$

and

$$
\omega_{2}=\gamma_{\mathrm{a}} / 2-\frac{1}{2}\left[\left(\gamma_{\mathrm{a}}^{2} / 4+\Delta^{2}+4 V^{2}+\gamma_{\mathrm{a}} \Delta\right)^{1 / 2}-\left(\gamma_{\mathrm{a}}^{2} / 4+\Delta^{2}+4 V^{2}-\gamma_{\mathrm{a}} \Delta\right)^{1 / 2}\right]
$$


where $\omega_{1}$ is the width of the line at $K=\frac{1}{2}\left(\Delta^{2}+4 V^{2}\right)^{1 / 2}$ and $\omega_{2}$ the width of the line at $K=-\frac{1}{2}\left(\Delta^{2}+4 V^{2}\right)^{1 / 2}$.

The two linewidths add up to $\gamma_{\mathrm{a}}$ (the linewidth of the single line when no coupling is considered). As $V$ increases, the linewidth $\omega_{1}$ decreases and $\omega_{2}$ increases. But an increase in $\Delta$ causes an increase in $\omega_{1}$ and decrease in $\omega_{2}$. Both linewidths approach $\gamma_{\mathrm{a}} / 2$ for large $V$. For $\Delta=0$, the two linewidths are equal and independent of $V$.

For $\Delta=\gamma_{\mathrm{a}}$, the probability $P\left(\Omega_{\mathrm{i}}, \hat{\boldsymbol{e}}_{\sigma}, \boldsymbol{k}_{\lambda}\right)$ is plotted in figure 3 as a function of $K$ for different $V$. For $V \neq 0$, a 'hole' appears at $K=-0.5 \gamma_{\mathrm{a}}$. This frequency is equal to the frequency difference between the non-decaying excited state and the ground state.

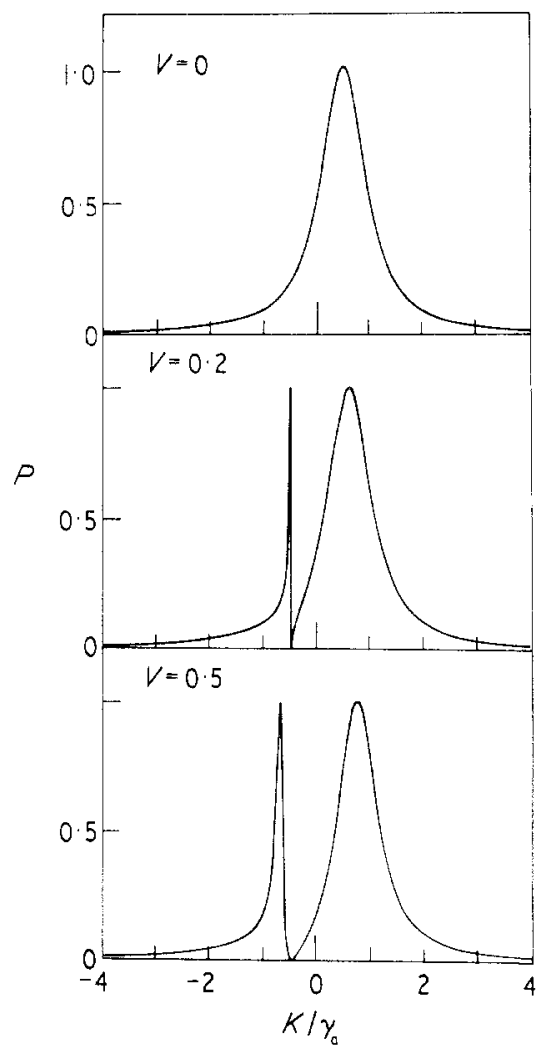

Figure 3. Frequency distribution of emitted radiation for the case when one of the excited states is non-decaying $\left(\gamma_{\mathrm{b}}=0\right)$. The energy difference $\Delta$ between the excited states is $\gamma_{\mathrm{a}}$. The origin of the $K$ axis is at the energy difference between the ground state and the average energy of the excited states.

The 'hole' in the emission line can be attributed to an interference phenomenon (Fontana and Srivastava 1973). One can solve for the eigenstates of the hamiltonian of the atom plus the external perturbation, in terms of the states $|a\rangle$ and $|b\rangle$. These perturbed eigenstates then interact with the radiation field. If one analyzes the relationship between the amplitudes of the two radiators, one finds that for large times the two interfere destructively at that frequency. 


\section{Resonance fluorescence with two decaying excited states with equal decay constants}

The probabilities as a function of time for this case $\left(\gamma_{a}=\gamma_{b}\right)$ have similar features as those discussed in $\S 3$, and thus the details are not discussed here. The probability $P\left(\Omega_{\mathrm{i}}, \hat{\boldsymbol{e}}_{\sigma}, \boldsymbol{k}_{\lambda}\right)$ that a photon with wavevector $\boldsymbol{k}_{\lambda}$ and polarization direction $\hat{\boldsymbol{e}}_{\lambda}$ has been emitted between time $t=0$ and $t \rightarrow \infty$ is obtained from equation (26) by setting $\gamma_{\mathrm{a}}=\gamma_{\mathrm{b}}=\gamma$ :

$$
\begin{aligned}
P\left(\Omega_{\mathrm{i}}, \hat{e}_{\sigma}, \boldsymbol{k}_{\lambda}\right)= & {\left[e^{4} I_{0} /\left(L^{3} \Gamma k_{0}^{3}\right)\right]\left[\left\{(K+\Delta / 2)^{2}+\gamma^{2} / 4\right\}\left|P_{\mathrm{ca}}^{\lambda}\right|^{2}\left|P_{\mathrm{ac}}^{\sigma}\right|^{2}\right.} \\
& +\left\{(K-\Delta / 2)^{2}+\gamma^{2} / 4\right\}\left|P_{\mathrm{cb}}^{\lambda}\right|^{2}\left|P_{\mathrm{bc}}^{\sigma}\right|^{2}+V^{2}\left\{\left|P_{\mathrm{ca}}^{\lambda}\right|^{2}\left|P_{\mathrm{bc}}^{\sigma}\right|^{2}+\left|P_{\mathrm{cb}}^{\lambda}\right|^{2}\left|P_{\mathrm{ac}}^{\sigma}\right|^{2}\right. \\
& \left.+4 \operatorname{Re}\left(P_{\mathrm{ca}}^{\lambda} P_{\mathrm{cb}}^{\lambda}\right) \operatorname{Re}\left(P_{\mathrm{ac}}^{\sigma} P_{\mathrm{bc}}^{\sigma *}\right)-2 \operatorname{Re}\left(P_{\mathrm{ca}}^{\lambda} P_{\mathrm{cb}}^{\lambda *} P_{\mathrm{ac}}^{\sigma} P_{\mathrm{bc}}^{\sigma *}\right)\right\} \\
& +2 V\left[\left\{(K+\Delta / 2)\left|P_{\mathrm{ca}}^{\lambda}\right|^{2}+(K-\Delta / 2)\left|P_{\mathrm{cb}}^{\lambda}\right|^{2}\right\} \operatorname{Re}\left(P_{\mathrm{ac}}^{\sigma} P_{\mathrm{bc}}^{\sigma *}\right)\right. \\
& +\left\{(K+\Delta / 2)\left|P_{\mathrm{ac}}^{\sigma}\right|^{2}+(K-\Delta / 2)\left|P_{\mathrm{bc}}^{\sigma}\right|^{2}\right\} \operatorname{Re}\left(P_{\mathrm{ca}}^{\lambda} P_{\mathrm{cb}}^{\lambda *}\right) \\
& +\gamma / 2\left\{\left(\left|P_{\mathrm{cb}}^{\lambda}\right|^{2}-\left|P_{\mathrm{ca}}^{\lambda}\right|^{2}\right) \operatorname{Im}\left(P_{\mathrm{ac}}^{\sigma} P_{\mathrm{bc}}^{\sigma *}\right)\right. \\
& \left.\left.+\left(\left|P_{\mathrm{bc}}^{\sigma}\right|^{2}-\left|P_{\mathrm{ac}}^{\sigma}\right|^{2}\right) \operatorname{Im}\left(P_{\mathrm{ca}}^{\lambda} P_{\mathrm{cb}}^{\lambda *}\right)\right\}\right] \\
& +\left(K^{2}-\Delta^{2} / 4+\gamma^{2} / 4\right) 2 \operatorname{Re}\left(P_{\mathrm{ca}}^{\lambda} P_{\mathrm{cb}}^{\lambda *} P_{\mathrm{ac}}^{\sigma} P_{\mathrm{bc}}^{\sigma *}\right) \\
& \left.+\gamma \Delta / 2 \operatorname{Im}\left(P_{\mathrm{ca}}^{\lambda} P_{\mathrm{cb}}^{\lambda *} P_{\mathrm{ac}}^{\sigma} P_{\mathrm{bc}}^{\sigma *}\right)\right]\left[\left\{(K-R)^{2}+\gamma^{2} / 4\right\}\left\{(K+R)^{2}+\gamma^{2} / 4\right\}\right]^{-1}
\end{aligned}
$$

where

$$
\begin{aligned}
R & =\frac{1}{2}\left(\Delta^{2}+4 V^{2}\right)^{1 / 2} \\
\Delta & =E_{\mathrm{a}}^{\prime}-E_{\mathrm{b}}^{\prime}
\end{aligned}
$$

and $K$ is defined in equation (34).

This probability is plotted in figure 4 as a function of $K$ for different $V$. Here, the matrix elements are assumed to be real and $P_{\mathrm{ca}}^{\lambda}=P_{\mathrm{cb}}^{\lambda}$ and $P_{\mathrm{ac}}^{\sigma}=P_{\mathrm{bc}}^{\sigma}$. In general, there are two peaks in the emission line, one near $K=R$ and the other near $K=-R$. An increase in $V$ suppresses the maximum near $K=-R$ and enhances the maximum near $K=R$. This effect is easily understood if one considers the eigenstates of the hamiltonian $H_{0}+V$ (excluding the radiation interaction). The two eigenstates can be written as:

$$
\begin{aligned}
& |1\rangle=a|a\rangle+b|b\rangle \\
& |2\rangle=b|a\rangle-a|b\rangle
\end{aligned}
$$

where

$$
\begin{aligned}
& a=\frac{1}{\sqrt{2}}\left(1+\frac{\Delta}{\left(\Delta^{2}+4 V^{2}\right)^{1 / 2}}\right)^{1 / 2} \\
& b=\frac{1}{\sqrt{ } 2}\left(1-\frac{\Delta}{\left(\Delta^{2}+4 V^{2}\right)^{1 / 2}}\right)^{1 / 2} \\
& V^{2}=|\langle a|V| b\rangle|^{2}
\end{aligned}
$$

and their corresponding energies are:

$$
\left.\begin{array}{l}
E_{1} \\
E_{2}
\end{array}\right\}=\left(E_{\mathrm{a}}^{\prime}+E_{\mathrm{b}}^{\prime}\right) / 2 \pm R .
$$




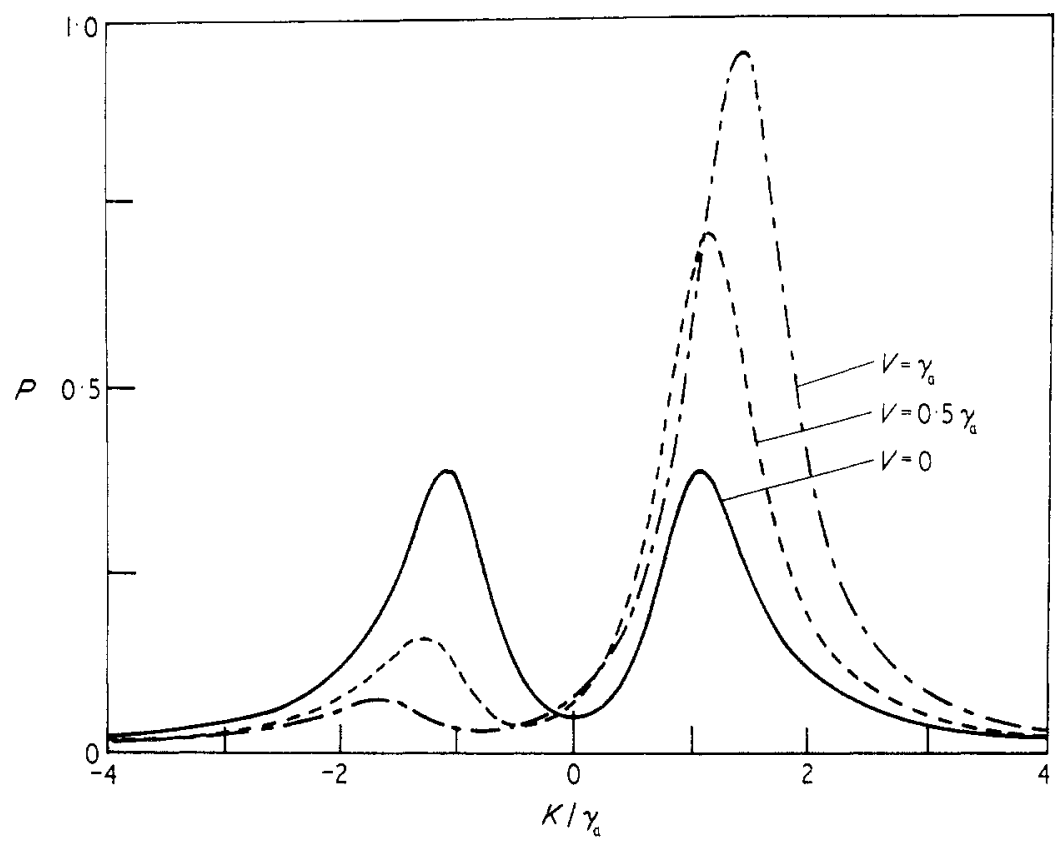

Figure 4. Frequency distribution of emitted radiation for the case when both excited states are decaying with the same rate $\left(\gamma_{\mathrm{a}}=\gamma_{\mathrm{b}}\right)$. The energy difference $\Delta$ between the excited states is $2 \gamma_{a}$. The origin of the $K$ axis is at the energy difference between the ground state and the average energy of the excited states.

For $E_{\mathrm{a}}^{\prime}>E_{\mathrm{b}}^{\prime}$, an increase in $V$ decreases $a$ and increases $b$. Both coefficients $a$ and $b$ approach $1 / \sqrt{ } 2$ for large $V / \Delta$. Thus as $V$ increases, the matrix element of the radiation interaction between the ground state and the eigenstate $|2\rangle$ tends to zero. Thus the peak near $K=-R$ is suppressed and disappears for large values of the static perturbation.

\section{References}

Barger R L and Hall J L 1969 Phys, Rev. Lett. 22 4-8

Bates D R 1961 Quantum Theory I. Elements (New York: Academic Press) p 203

Bethe H A and Salpeter E E 1957 Quantum Mechanics of One- and Two-Electron Atoms (Berlin: SpringerVerlag)

Breit G 1933 Rev. mod. Phys. 591-140

Brewer R G, Kelly M T and Javan A 1969 Phys. Rev. Lett. 23 559-63

Fontana P R and Srivastava R P 1973 Phys. Rev. A 7 1866-9

Hansch T W, Levenson M D and Schawlow A L 1971 Phys. Rev. Lett. 26 946-9

Heitler W 1960 The Quantum Theory of Radiation (London: Oxford University Press)

Himmell L C 1965 Thesis, University of Michigan

Lee P H and Skolnick M L 1967 Appl. Phys. Lett. 10 303-5

Srivastava R P 1972 Thesis Oregon State University

Weisskopf V and Wigner E 1930 Z. Phys. 63 54-73; 65 18-29 